\title{
Archéologie des représentations
}

Jean-François Battail

Université Paris-Sorbonne

Depuis la fin de la Seconde Guerre mondiale, les échanges culturels entre la France et la Suède se sont multipliés, tant sur le plan institutionnel que par le biais de réseaux informels. L'essor des techniques de communication, en premier lieu l'avènement de l'informatique, a rendu possible ce resserrement des liens. D'où l'intérêt du présent colloque destiné à faire le point sur les médiations, trajectoires et circulations entre nos deux pays de I945 à nos jours. Quelles que soient les évolutions, de nombreuses représentations toujours vivaces plongent leurs racines parfois très loin dans le temps. Il ne paraît donc pas inutile, en guise de préambule, d'évoquer le passé pour mieux appréhender le présent. Sans prétendre résumer en quelques pages l'histoire de nos relations au fil des siècles, les remarques qui suivent ne visent qu'à cerner un certain nombre de traits qui d'une façon ou d'une autre affleurent encore aujourd'hui.

Nos représentations mutuelles s'inscrivent dans un contexte plus vaste, celui de la confrontation Nord-Sud qui remonte à l'Antiquité et n'a cessé de se manifester sous des formes diverses au cours de toute l'histoire européenne. La dichotomie romain-barbare, renforcée par l'immémoriale théorie des climats, a donné naissance à une géographie de l'imaginaire qui a connu de nombreux avatars. Certes, la recherche moderne a montré qu'il y eut des rapports plus étroits que l'on ne l'imaginait autrefois entre l'empire romain et le monde germanique. Et la culture latine, avec les éléments grecs et judéo-chrétiens qu'elle véhiculait, a progressivement gagné le Nord du continent. Depuis un bon millénaire, le Nord est inclus dans l'Europe culturelle, il repose sur un socle que nous avons en commun. Cependant, à l'Antiquité classique et la latinité chrétienne s'ajoute une dimension proprement nordique, un

How to cite this book chapter:

Battail, J.-F. 20I 5. Archéologie des représentations. In: Cedergren, M. et Briens, S. (eds.) Médiations interculturelles entre la France et la Suède. Trajectoires et circulations de I945 à nos jours. Pp. 3-I 5. Stockholm: Stockholm University Press. DOI: http://dx.doi. org/Io.I6993/bad.a. License: CC-BY 
vigoureux héritage norrois qui lui confère une identité propre. Même si nous sommes tributaires les uns et les autres d'un même patrimoine qui tend à gommer les différences, il faut aussi tenir compte de ce facteur spécifique.

Dans l'imaginaire collectif, le Nord et le Midi n'ont jamais été dotés de frontières bien précises. Pour Voltaire, la Russie fait partie du Septentrion au même titre que les pays nordiques au sens où nous l'entendons aujourd'hui, et Mallet, son contemporain, amalgame Scandinaves et Celtes dans un célèbre ouvrage qui a fait date ${ }^{\mathrm{I}}$. Nous nous limiterons ici à la Suède dans le rapport qu'elle a entretenu avec la France. Non sans quelque arbitraire, car les liens culturels ne se réduisent jamais à des relations bilatérales. D'abord, la Suède ne peut être dissociée de son contexte scandinave. Si les Suédois et les Danois se sont longtemps considérés comme des ennemis héréditaires, ils ont eu en même temps clairement conscience de partager un héritage culturel commun - qu'ils se sont d'ailleurs âprement disputés ${ }^{2}$. En second lieu, quand on parle d'apports français à la culture suédoise, il n'est pas toujours aisé de les distinguer d'autres éléments importés, notamment d'Italie. Et l'Allemagne a souvent servi de trait d'union entre la Scandinavie et le monde roman, comme l'a rappelé opportunément un ouvrage récent consacré au triangle franco-germano-nordique ${ }^{3}$. Les influences culturelles s'exercent rarement de manière linéaire. Quand les Scandinaves découvrent l'œuvre philosophique de Voltaire, c'est la pensée de Newton et de Locke qui vient à eux en habits français. C'est en traduction française que Dalin découvre Addison et Steele, inspirateurs de son propre Argus, que Kellgren s'ouvre à la vague ossiannique qui fait passer un frisson nouveau dans la poésie européenne. On pourrait invoquer bien d'autres exemples. Cela dit, il est parfaitement légitime de se limiter à une perspective franco-suédoise pourvu qu'on garde à l'esprit qu'elle s'inscrit toujours dans un contexte plus large.

Chronologiquement, on peut distinguer trois grandes phases dans la manière dont le Nord s'est profilé par rapport au Midi. Le premier millénaire est celui des barbares, des vikings, avec les premières tentatives de christianisation à l'époque carolingienne, même si "l'apôtre du Nord ", Anschaire (Ansgar), n'a eu qu'une influence limitée dans le Septentrion. Une évolution graduelle s'observe néanmoins. Avec la fondation du duché de Normandie en 9i I (traité de Saint-Clair sur Epte), les hommes du Nord qui avaient semé la terreur dans le royaume franc reçoivent le baptême et deviennent des administrateurs modèles. Baptisé à Rouen, le jeune Olav Haraldsson, futur saint Olav, tirera du 
reste profit de son expérience normande pour jeter les bases de l'État norvégien au début du deuxième millénaire.

Vient ensuite, en ce qui concerne la Suède, l'intégration progressive dans la sphère catholique et l'orbe latine. Processus entièrement accompli au XIII siècle à l'époque des Folkungar où sainte Brigitte apparaît comme la première personnalité suédoise de rayonnement international. La Sorbonne attire alors les étudiants suédois et l'on voit apparaître sur la Montagne Sainte-Geneviève des collèges (Uppsala, Skara et Linköping) destinés à les accueillir. Quand plus tard sera créée en I 477 l'université d'Uppsala, la première université scandinave, celle-ci adoptera les statuts de la Sorbonne sous l'impulsion de son promoteur Jacob Ulvsson, lui-même formé à Paris.

L'universalité du monde catholique et latin va être rompue avec l'instauration du luthéranisme par Gustave Vasa au XVI e siècle, et c'est la troisième phase. De vives tensions s'ensuivent entre catholiques et protestants, mais il existe malgré tout des passerelles du fait de la complémentarité entre nos deux pays. Le climat a beau être infecté, les intérêts politiques, commerciaux et militaires priment - François ${ }^{\text {er }}{ }^{\text {et Gustave }}$ Vasa signent ainsi un traité de coopération en I 542. Et au siècle suivant, ne voit-on pas la France catholique de Richelieu s'allier à la Suède protestante de Gustave-Adolphe lors de la guerre de Trente Ans pour combattre un ennemi commun, les Habsbourg. Un fossé culturel s'est néanmoins créé, et l'on en ressent les effets jusqu'à nos jours, non sous forme directement confessionnelle, mais en matière de valeurs et de comportements, fruits d'une longue imprégnation pédagogique. En voici une illustration, anecdotique sans doute, mais néanmoins révélatrice. Quand l'île de Saint-Barthelémy, un temps suédoise, fut cédée à la France en I 878, un article paru dans Ny illustrerad tidning le 27 avril de cette même année rendit compte des cérémonies officielles en insistant sur les tensions religieuses et les différences de mentalité. Outre qu'il dénonce les menées du clergé catholique toujours prompt à agir contre le protestantisme, l'auteur insiste sur la manière dont se manifestent les caractères nationaux. À l'occasion des festivités, notamment d'une régate, il est frappé par l'agitation, les cris, les plaisanteries, les grands gestes et éclats de rires qui caractérisent les Français, enclins à ne voir en toute chose que réjouissance (en français dans le texte) - et de noter par contraste la dignité des compétiteurs suédois qui ne voient que le côté sérieux des choses ; ni bavardage ni exclamations ici, et un sens des responsabilités qui ne se dément jamais. Caricature ? Stéréotypes complaisants? Certaines constantes ont en tout cas la vie dure, et bon 
nombre d'éléments qu'on pourrait juger archaïques se retrouvent au cœur de la modernité. Dans nos relations interculturelles, les incompréhensions ou reproches mutuels sont souvent du même type. Côté suédois, on se prévaut de rationalité, de pragmatisme, de maîtrise de soi, et l'on accorde un rôle positif au silence, synonyme de réflexion. Et l'on reproche volontiers aux Français, souvent désarçonnés par la façade trop lisse de leurs interlocuteurs, leur goût immodéré pour la rhétorique, une certaine forme d'élitisme et une tendance au désordre et à l'anarchie. D'où dans le pire des cas une accusation mutuelle d'arrogance, mais pour des raisons strictement inverses.

Malgré tout, nos relations à travers les âges ont été presque toujours au beau fixe, ce qui justifie qu'une anthologie qui leur a été consacrée ait pu s'intituler Une Amitié millénaire 4 . Aucune guerre n'a mis aux prises nos deux pays, si l'on excepte quelques offensives françaises en Poméranie suédoise lors des guerres napoléoniennes. Et le fait qu'un des chefs militaires impliqués, Bernadotte, ait été élu quatre ans plus tard héritier de la couronne suédoise, permet d'en relativiser la portée. Il demeure qu'il y eut des périodes plus ou moins fastes en fonction des fluctuations de la conjoncture politique. La Réforme a entrainé un refroidissement des relations, tout comme la Révolution française. Gustave III avait beau avoir applaudi Beaumarchais à Paris quelques années auparavant, il prit résolument la tête d'une croisade contre-révolutionnaire, effaré que les « charmants Français » soient devenus " les orangs-outans de l'Europe ». Sans doute, quelques intellectuels comme Kellgren et Thorild ont salué les événements de I789, mais la Terreur a bien vite refroidi les enthousiasmes. Au cours des années qui ont suivi, il ne faisait pas bon être soupçonné de jacobinisme en Suède. Il demeure que l'influence française a été grande à partir du XVII ${ }^{e}$ siècle jusqu'à la guerre franco-prussienne de I870, date à laquelle la Suède officielle s'est plutôt orientée vers l'Allemagne. Cette influence a connu son apogée au XVIII e siècle, du moins dans les hautes classes, et non sans résistances. Dans l'autre sens, des impulsions en provenance de Suède (et de Scandinavie) se sont manifestées en France par vagues successives. Sans remonter à sainte Brigitte, mentionnons Linné au XVIII ${ }^{e}$ siècle, son contemporain Swedenborg à titre posthume, mais avec un impact d'autant plus grand chez nos romantiques, Tegnér et Geijer dans les années I 830-I840, la mythologie nordique (re)découverte qui inspire notamment les Poèmes barbares de Leconte de Lisle, et surtout les écrivains de la percée moderne, Strindberg et Ibsen en tête, de I 890 à la Première Guerre mondiale, sans oublier Selma Lagerlöf, première 
femme à obtenir le prix Nobel de littérature (I909). L'importance du facteur culturel apparaît capitale car on peut constater que les refroidissements passagers sur le plan politique n'ont jamais affecté en profondeur la qualité et l'intensité des échanges entre artistes, intellectuels et scientifiques de nos deux pays.

En termes d'images, le Nord vu du Sud révèle un mélange constant de fascination et d'inquiétude. Dans Germania (98 ap. JC), Tacite, luimême tributaire d'Aristote, avait déjà donné le ton en décrivant, sur la base de témoignages de voyageurs, des peuples guerriers et pourtant épris de paix, des barbares civilisés en quelque sorte. Leur brutalité, indéniable, allait de pair avec le respect de la loi et le goût de l'ordre. Cette image paradoxale d'un Nord arcadien, idyllique à bien des égards, mais rude et peu civilisé, allait connaître une fortune remarquable. Selon les époques et les contextes, l'une de ces deux facettes, Barbaria ou Utopia, allait être mise en exergue, mais sans que l'autre, simplement refoulée, disparaisse totalement. Les jugements portés sur les Vikings révèlent cette dualité.

Plus près de nous, et pour nous en tenir à la Suède, n'oublions pas qu'elle a été un redoutable État militaire à l' " Ère de grande puissance ", depuis le règne de Gustave-Adolphe jusqu'à la mort de Charles XII. Rentrée dans le rang après I7I 8 , elle a compensé ses pertes territoriales par un remarquable essor à l'intérieur même de ses frontières, notamment sur le plan scientifique. Malgré cette reconversion pacifique, des échos guerriers n'allaient cesser de retentir par la suite, ne seraitce qu'en sourdine. Linné, l'un des grands artisans du rayonnement intellectuel de la Suède à l' " ère de la liberté ", affectionnait les métaphores militaires; commandant en chef des naturalistes, il envoyait ses disciples à la conquête du monde pour la plus grande gloire de sa patrie, et bon nombre de ces apôtres-soldats l'ont payé de leur vie. Les grands explorateurs de la seconde moitié du XIX siècle, comme Nordenskiöld, apparaissaient aux yeux de l'opinion publique comme des vikings pacifiques. Quand à partir de la fin du XIX ${ }^{\mathrm{e}}$ siècle le pays s'est imposé comme grande puissance industrielle grâce à une pléiade d'inventeurs et d'innovateurs (on a parlé à ce sujet d'industrie du génie, "snilleindustri »), Gerhard de Geer s'est cru autorisé à parler de "Deuxième âge de grandeur de la Suède » (I928), allumettes, roulements à billes et centrifugeuses se substituant aux armes des Carolins's. Même l'hymne national suédois, pourtant si paisible et d'inspiration romantique, comporte une allusion aux jours glorieux de l'ère de grande puissance. Nul n'incarne mieux cette ambivalence qu'Alfred Nobel, Suédois célèbre s'il 
en est : l'idéaliste convaincu, le créateur du prix de la Paix, a été toute sa vie fasciné par les armes, et ses inventions ont contribué à renforcer le potentiel destructeur de l'humanité, même si ses intentions étaient tout autres. Janus au double visage ${ }^{6}$ ?

Mais l'autre image, tout aussi prégnante et tout aussi ancienne, est celle d'un Nord vertueux où pureté de la nature et rectitude morale vont de pair — thème qui resurgit à intervalles réguliers dans l'histoire des idées. Dans le domaine francophone, le moment crucial se situe à l'âge des Lumières lorsque Tacite est l'objet d'une réinterprétation politique. Relayant des sources nordiques (Olaus Magnus en premier lieu, mais aussi Olof Rudbeck qu'il qualifie de " célèbre »), Montesquieu exalte l'amour de la liberté de ces nations vaillantes qui s'opposent à la tyrannie. « Les peuples du Nord de l'Europe se maintinrent dans une sagesse admirable contre la puissance romaine jusqu'au jour où ils sortirent de leurs forêts pour la détruire ». C'est sous ces latitudes septentrionales qu'il situe le berceau de la liberté en Europe, et cette caractéristique rejaillit sur les Francs, d'origine germanique. Il s'agit en quelque sorte de s'approprier la Scandinavie, ou du moins de l'ériger en modèle. L'auteur de l'Esprit des Lois est une source majeure d'inspiration pour PaulHenri Mallet, historien genevois au service du roi de Danemark, traducteur des Eddas, grand vulgarisateur au rayonnement européen. Voltaire, dans l'Essai sur les moeurs, présente la Suède comme le royaume de la terre le plus libre. L'Encyclopédie de Diderot et d'Alembert, avec entre autres les contributions de Jaucourt et le long article "Edda » signé d'Holbach, confèrent une place centrale dans le débat politique et culturel à ce Septentrion qui jusqu'alors n'avait eu qu'une position périphérique, et ce, paradoxalement, à une époque où une gallomanie parfois sans nuance fait des ravages parmi les élites scandinaves. Le ton était donné. Élu héritier du trône de Suède, Bernadotte, lui-même architecte de la pax scandinavica, rend hommage dans sa correspondance avec Mme de Staël à cet amour de la liberté qu'il avait trouvé, disait-il, inné chez les Suédois. Gobineau, ambassadeur de France à Stockholm de I 872 à I 877 , se fait l'écho de cette vision idyllique dans ses lettres et brosse le portrait d'un société préfigurant de manière saisissante ce qu'on appellerait beaucoup plus tard le "modèle suédois ${ }^{7}$ ". Nature, liberté, liberté naturelle, autant de concepts qui jouent un rôle central dans l' " utopie ambiguë» — finement analysée par Vincent Fournier —

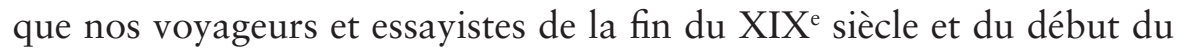
$\mathrm{XX}^{\mathrm{e}}$ ont illustrée ${ }^{8}$. Le stéréotype arcadien d'une société pacifiée s'inscrit donc dans une longue tradition; en ce sens, le folkhem qui allait prendre 
forme dans les années I930 marque tout autant un aboutissement que l'avènement d'une nouvelle ère.

Le Sud vu du Nord apparaît lui aussi sous des formes contrastées. Peu peuplés, soumis aux aléas d'un climat rude, les pays nordiques ont été tributaires d'impulsions venus de l'extérieur. Des vagues successives ont enrichi leur culture et, pour s'en tenir aux temps modernes, on peut distinguer diverses phases au cours desquelles des apports français, allemands, anglo-saxons se sont greffés sur un vieux substrat autochtone ; l'Italie et les Pays-Bas, entre autres, ont également été sources d'inspiration dans divers domaines. Cette dépendance à l'égard de l'étranger a parfois incité les intellectuels scandinaves à minimiser leur propre culture, comme si elle se réduisait à la somme des influences reçues. Et beaucoup d'observateurs suédois, passés maîtres dans l'autocritique, ont dénoncé chez leurs compatriotes une tendance à imiter ce qui venait d'ailleurs, comme si l'herbe était plus verte chez le voisin. À quoi s'ajoute le fait que les Scandinaves, faute de pouvoir se faire comprendre hors de leurs frontières, ont dû s'initier aux langues étrangères, ce qui leur a permis de s'ouvrir à d'autres modes de pensée et expressions culturelles. D'où un cosmopolitisme qui a contribué à valoriser le rôle civilisateur des cultures venues du Sud.

Cependant, des périodes de repli sur soi ont ponctué l'histoire à intervalles réguliers. On a parlé à ce sujet de "Renaissances nordiques » (Anton Blanck) ou encore de "primitivisme nordique » (Tore Frängsmyr). Le göticisme, aux racines très anciennes, peut être vu comme une affirmation identitaire tendant à exalter les vertus supposées des ancêtres nordiques au détriment des peuples du Midi. L'irruption des hommes du Nord dans l'Empire romain était vue en ce sens comme une injection de vitamines dans une civilisation déliquescente. La force et la fécondité étaient l'apanage du Septentrion — au VIe siècle déjà, Jordanes présentait la patrie des Gots comme " matrice des peuples, creuset des nations » ("vaginam nationum, officina gentium »), tout comme la simplicité des mœurs et la rectitude morale. Cette glorification du Nord vivifiant au détriment du Sud émollient a connu en Suède son apogée aux $\mathrm{XVI}^{\mathrm{e}}$ et XVII ${ }^{\mathrm{e}}$ siècles. Sans entrer dans le détail, mentionnons l'apport majeur de Johannes Magnus à l'époque de Gustave Vasa ; l'on doit à ce prélat en exil une monumentale chronique en latin des souverains "suédois» (Historia de omnibus gothorum sveonumque regibus, posth. I 554 ) qui, à l'aide d'une chronologie largement fantaisiste, tend à montrer que la Suède est le plus ancien royaume du monde — il remonterait à Magog, le petit-fils de Noé ! Le fait qu'Erik XIV, fils aîné et succes- 
seur de Gustave Vasa, ait traduit cet ouvrage en suédois montre bien l'utilisation politique qu'on pouvait en faire à une époque où la dignité d'un royaume se mesurait à l'aune de son ancienneté. Ainsi revisité, le vieux mythe n'allait cesser de s'enrichir d'éléments nouveaux. Quelques décennies plus tard, quand la Suède accédait au rang de grande puissance, il importait de forger une identité nationale digne de l'ère de grandeur ! Le programme officiel de l'administration des Antiquités nationales créé par Gustave-Adolphe en I629 n'était autre que le göticisme. Le peuple suédois était le plus ancien au monde, et le suédois la langue-mère de l'humanité. L'exaltation gotique allait atteindre son point d'incandescence avec l'ouvrage monumental et pourtant inachevé d'Olof Rudbeck, Atland eller Manhem (Atlantica) - quatre volumes en suédois avec texte latin en regard (I679-I702) - qui redonnait vie à la vieille légende des Hyperboréens et affirmait que l'Atlantide prétendument engloutie qu'évoque Platon n'était autre que sa propre patrie à laquelle il entendait redonner tout son lustre aux yeux du monde saisi d'admiration. Il est caractéristique que cet ouvrage aussi génial que monstrueux ait été financé par le très officiel Collège des Antiquités (Antikvitetskollegium)'.

$\mathrm{Au}$ XVIII ${ }^{e}$ siècle, ces rêveries gotiques allaient être largement déconstruites sous l'influence du rationalisme critique régnant; subsistait néanmoins le thème primitiviste attribuant aux ancêtres l'innocence et la simplicité des mœurs liées à une vie proche de la nature. Et le mythe göticiste des vertueux Gots opposés aux Romains cultivés mais corrompus était largement repris et légitimé par des étrangers comme Montesquieu et Mallet, mentionnés précédemment, mais aussi l'Anglais Percy, l'Allemand Herder - à une époque où les modèles français étaient dominants en Europe !

En matière de regards croisés, le XVIII ${ }^{e}$ siècle offre d'intéressantes perspectives. À l'heure où tant d'intellectuels de l'Ancien Continent découvraient les vertus du Nord, la langue et la culture françaises poursuivaient leur percée victorieuse dans le nord de l'Europe et spécialement en Suède. Amorcé au XVI siècle, le mouvement s'était amplifié à l'époque de la grande puissance suédoise. Les jeunes nobles devaient être éduqués à la française. L'université d'Uppsala créa en I637 un poste de maître de langues, le tout premier de ce genre en Suède, et par langues, il fallait entendre le français et, à un degré moindre, l'italien. Le règne de la reine Christine renforça encore ce type d'influence, à tel point qu'un poète patriote comme Stiernhielm, lui-même polyglotte chevronné et parfaitement intégré à la Cour, ressentit la nécessité de se faire l'apôtre 
de la langue suédoise — « langue de l'honneur et des héros » («ärans och hjältarnas språk ${ }^{\text {זo } ») ~ S i ~ t e l ~ o u ~ t e l ~ c o n c e p t ~ f a i s a i t ~ d e ́ f a u t ~ d a n s ~ l a ~}$ langue nationale, le "père de la poésie suédoise » préconisait de les forger à partir des vieilles racines norroises plutôt que de les emprunter à l'étranger. Une résistance assez farouche s'organisa ainsi, et parmi les tenants de cette fierté hyperboréenne, certains se servirent du français pour mieux vilipender la France et ses dérisoires petits-maîtres, comme le révèle une excellente anthologie récemment parue à Stockholm. ${ }^{\text {II }}$ Anders Kempe alla jusqu'à écrire en I688 : "Dieu parle suédois [...] et le serpent parle français » - il fallait oser, même si le propos de l'auteur n'est pas dépourvu d'ironie ${ }^{\mathrm{I} 2}$ ! Jusqu'à la Révolution française et même au-delà, cela n'empêcha pas la poursuite de la francisation des hautes classes, Gustave III en tête. C'est l'époque où se constitua un véritable cosmopolitisme lexical du fait des nombreux emprunts qui vinrent enrichir la langue suédoise. Enrichir ou dénaturer ? Véritable cheval de Troie, la langue importée ne véhiculait-t-elle pas aussi des modes de pensée exogènes qui menaçaient de subvertir la culture autochtone ? Beaucoup s'en émurent, y compris parmi ceux qui se devaient de maîtriser le français, tel le poète de cour Olof Dalin. Sur l'échiquier politique, les Bonnets, partisans d'une morale bourgeoise d'inspiration anglo-saxonne, se montrèrent beaucoup plus rétifs à la gallomanie ambiante que leurs rivaux les Chapeaux. Quant au clergé, il ne cessa de s'élever contre la dissolution des mœurs dont la culture française était censée être le vecteur. Les sociétés savantes se mobilisèrent aussi pour la défense et l'illustration de la langue maternelle, l'Académie des sciences d'abord, fondée en I739, puis l'Académie des Belles-Lettres de LouiseUlrique et enfin l'Académie suédoise créée en I786 par Gustave III sur le modèle français, certes, mais dont les missions étaient résolument nationales. Il serait donc exagéré de parler d'hégémonie du français, d'autant qu'à l'époque, la population suédoise était rurale à $90 \%$.

La perte de la Finlande provoqua un nouveau sursaut patriotique, en pleine tourmente des guerres napoléoniennes. Dès I 8 I I, les poètes regroupés au sein de la société Göthiska förbundet, Geijer et Tégner en tête, firent opportunément ressortir les Vikings de leurs tombes. L'heure n'était plus au göticisme monumental d'un Rudbeck, mais plutôt à une mobilisation patriotique qui, sur arrière-fond de désastre politique, entendait rappeler les vertus des ancêtres — amour de la liberté, courage viril et droiture morale. Le poème de Geijer, "Vikingen ", parut en I8 I I dans le premier numéro d'Iduna, la revue de la société. Traduit ultérieurement en français, il allait faire grande impression 
sur nos compatriotes, de même que Frithjofs saga de Tegnér, véritable best-seller chez nous ${ }^{\mathrm{I}}$. Si Geijer se montrait plus que réticent à l'égard de la culture française, le classicisant Tegnér, au contraire, en recevait des impulsions fondamentales, ce qui n'altérait en rien sa fibre patriotique. Mais l'on peut aussi noter que son Viking, Frithjof, ressemble plus à un ancien Grec qu'à un pillard des mers, ce que d'aucuns, notamment en France, n'ont pas manqué de lui reprocher. Dans un essai génial ayant pour thème la pauvreté suédoise, Svenska fattigdomens betydelse (I 838), Almqvist opposait pour sa part la culture des manoirs (berrgårdskultur) à la culture paysanne traditionnelle (allmogekultur), la seule authentiquement suédoise à ses yeux. À la fin du XIX ${ }^{\mathrm{e}}$ siècle, sous l'impulsion d'un romantisme national tardif, le style néo-gothique s'imposait un temps alors que simultanément, la création de Skansen et autres musées de plein air avait pour but, à l'heure de l'industrialisation, de préserver l'authentique culture populaire.

Le courant göticiste aurait-il disparu à l'ère de la construction européenne et de la mondialisation ? Rien n'est moins sûr. À cet égard, une analyse fine des débats et discussions en Suède entre pro- et antieuropéens lors de différentes consultations populaires mettrait certainement en lumière la persistance de ce clivage Nord-Sud que les différences confessionnelles ont renforcé à partir de la Réforme. En vérité, il y a toujours eu deux Nord et deux Sud, chacun affecté d'un signe positif ou négatif selon les besoins de la cause. Nos regards mutuels ont valeur de tests projectifs. Quand nos philosophes des Lumières rêvent de liberté, le Nord devient paradigmatique. Quand les écrivains nordiques de la " percée moderne » fantasment sur la Renaissance, c'est en réaction contre le moralisme et le conformisme étriqué qu'ils dénoncent dans leur propre société. L'ailleurs devient ainsi le lieu mythique de nos rêves et parfois de nos angoisses.

Au total, nos regards croisés révèlent un intérêt mutuel et une charge affective qui s'expliquent par le fait que nous allons chercher chez l'autre ce qui nous paraît manquer chez nous. Si tension il y a, elle se révèle productive car la distance qui nous sépare est suffisamment limitée pour que nous puissions nous enrichir de nos différences. Le maître-mot est ici celui de complémentarité. Les Suédois qui se sont rendus en France au fil des siècles étaient avant tout attirés par les milieux urbains - la capitale en premier lieu, ou encore telle ou telle université où l'on pouvait parfaire ses études ou obtenir un grade académique. Parlant de Paris, Nobel disait plaisamment que le moindre 
roquet y respirait la civilisation (" här luktar civilisation om varenda hundräcka » $)^{\mathrm{I}}$. Inversement, c'est la nature suédoise et l'ingéniosité des hommes à en tirer parti qui attirait les voyageurs du Nord. Stockholm n'était qu'une étape vite franchie par les Français qui, du romancier Regnard (qui avait atteint Jukkasjärvi en I68I) au géographe Rabot (auteur de la première escalade du mont Kebnekaise en I883), ont toujours témoigné d'un goût prononcé pour ce "Nord qui touche aux confins de la terre vivante " (le mot est de Mme de Staël). Nos voyageurs n'auraient manqué pour rien au monde la grande mine de cuivre de Falun, au demeurant source d'inspiration littéraire pour l'abbé Prévost et le marquis de Sade (sans oublier E. T. A. Hoffmann). Peu peuplée, riche de terres encore vierges, la Suède présentait un contraste saisissant avec l'hypercivilisation des salons parisiens tout en offrant de nombreux exemples d'adaptations réussies à un difficile environnement naturel. Une telle disparité entre nos deux pays explique en partie la nature de leurs échanges. Cela s'observe notamment dans le domaine scientifique. Alors que les Français excellaient dans les disciplines les plus abstraites, les mathématiques en premier lieu, les Suédois allaient développer au contact de la nature un savoir en prise directe avec les réalités concrètes - une nécessité dans un pays où, comme le disait le génial inventeur Christopher Polhem, "l'on doit plus penser à son pain quotidien qu'à des curiosités $»^{15}$.

Les échanges scientifiques à l'âge des Lumières apparaissent typiques d'un enrichissement mutuel fondé sur la complémentarité mais l'on pourrait invoquer des exemples tout aussi frappants dans d'autres domaines tels que la littérature ou les beaux-arts. Le déséquilibre démographique a parfois incité des commentateurs pressés à ne considérer les échanges intellectuels entre les deux pays que sous un angle purement quantitatif, comme s'il s'agissait de marchandises soumises à la douane. À ce jeu, le plus gros est toujours sûr de l'emporter ! Certains ont même eu recours sans nuance au modèle réducteur d'un centre qui rayonne et d'une périphérie qui en reçoit les effets... Quiconque s'intéresse aux relations culturelles franco-suédoises sait à quel point de telles représentations sont fallacieuses. Les éléments venus du dehors ont toujours été filtrés, adaptés, transmutés, et les exemples d'hybridations réussies ne manquent pas. D'où un équilibre dans nos relations qui explique sans doute l'estime réciproque dans laquelle nous nous tenons, et par voie de conséquence, le caractère nettement positif des représentations mutuelles qui ont jalonné l'Histoire. 


\section{Notes}

I. Mallet, P. H. (I756), Monuments de la Mythologie et de la Poésie des Celtes et particulièrement des Anciens Scandinaves, Copenhague.

2. Voir à ce sujet Battail, J.-F. (I992), "Entre Réforme et Romantisme» in Battail, J.-F., Boyer R., \& Fournier, V., Les Sociétés scandinaves de la Réforme à nos jours. Paris : PUF : 90-IO2 et I60-I72.

3. Espagne, M. (éd.) (2006), Le prisme du Nord. Pays du Nord, France, Allemagne. Tusson.

4. Battail, M. \& J.-F. (éd.) (I993), Une Amitié millénaire. Les relations entre la France et la Suède à travers les âges. Paris : Beauchesne.

5. Voir Kylhammar, M. (2004), "Sveriges andra stormaktstid », in Den tidlöse modernisten, Stockholm : Carlssons : 99- I 8. Traduction française de Battail, M. \& J.-F. (2009), Le Moderniste intemporel. Paris : L'Harmattan.

6. Sur ce thème, voir Battail, J.-F. (2007), "Le Nord aux deux visages ", in Andersson, K. (éd.), L'Image du Nord chez Stendhal et les Romantiques IV. Örebro : 72-88; également du même auteur (2012), "L'Image du Nord en France et les études scandinaves ", in Cent Ans d'études scandinaves. KVHAA konferenser 77. Stockholm : 85-100.

7. Voir Donnard, J.-H. ( I988), "Gobineau et le modèle suédois », in Proschwitz (von), G. (éd.), Influences. Göteborg, Paris ; également, Gobineau (de), A. (I994), Correspondance diplomatique I872-I877. Ed. de Raymond (de), M. \& J.-F., Paris : Touzot.

8. Fournier, V. (I989), L’Utopie ambiguë. La Suède et la Norvège chez les voyageurs et essayistes français (I882-I9I4). Clermont-Ferrand : Adobe.

9. Pour une approche plus détaillée du courant göticiste, voir Battail, J.-F. (2000), "Le Nord triomphant ", in Dubar M. et Moura J.-M. (éd.) (2000), Le Nord, latitudes imaginaires. Lille : Université Charles de Gaulle : 25-34.

Io. Tegnér, E. (I8I7), "Språken ». Mindre dikter. Project Runeberg http:// runeberg.org/tegner/057.htlm

I I. Östman, M. \& H. (2005), Au champ d'Apollon. Ecrits d'expression française produits en Suède (I 50-2006). Stockholm : Kungl. Vitterhetsakademien. I2. Cité par Östman, M. \& H. (2005 : 20). Des mêmes auteurs, voir aussi (2006), "Admiration et aversion. L'attitude des Suédois à l'égard des Français aux XVII ${ }^{\mathrm{e}}$ et XVIII ${ }^{\mathrm{e}}$ siècles ». Études Germaniques 4 : 229-246.

I3. Voir Gravier, M. (I943), Tegnér et la France. Paris: Aubier.

I4. Cité par Fant, K. (I995), Alfred Bernhard Nobel (2e éd.). Stockholm : Norstedts : I34.

I 5 . Dans Patriotiska testamentet (posthume, I76I) : « ... ett land, där var måste tänka mer på brödfödan än på curieusiteter », ch. I $8: 78$. 


\section{Bibliographie}

Battail, J.-F. (I992), « Entre Réforme et Romantisme » in Battail, J.-F., Boyer R., \& Fournier, V., Les Sociétés scandinaves de la Réforme à nos jours. Paris : PUF : 90-102 et I60-I72.

Battail, M. \& J.-F. (éd.) (1993), Une Amitié millénaire. Les relations entre la France et la Suède à travers les âges. Paris: Beauchesne.

Battail, J.-F. (2000), « Le Nord triomphant », in Dubar M. et Moura J.-M. (éd.), Le Nord, latitudes imaginaires. Lille : Université Charles de Gaulle : 25-34.

Battail, J.-F. (2007), "Le Nord aux deux visages ", in Andersson, K. (éd.), L'Image du Nord chez Stendhal et le Romantiques IV. Örebro : 72-88.

Battail, J.-F. (20I2), "L'Image du Nord en France et les études scandinaves ", in Briens, Gadelii, Lehman et Maillefer (éd.), Cent Ans d'études scandinaves. KVHAA konferenser 77. Stockholm : 85-100.

Donnard, J.-H. (I988), " Gobineau et le modèle suédois » in Proschwitz (von), G. (éd.), Influences. Göteborg, Paris.

Espagne, M. (éd.) (2006), Le prisme du Nord. Pays du Nord, France, Allemagne. Tusson.

Fournier, V. (1989), L'Utopie ambiguë. La Suède et la Norvège chez les voyageurs et essayistes français (I882-I9I4). Clermont-Ferrand : Adobe.

Gobineau (de), A. (1994), Correspondance diplomatique I872-1877. Ed. de Raymond (de), M. \& J.-F., Paris : Touzot.

Gravier, M. (I943), Tegnér et la France. Paris: Aubier.

Kant, K. (1995), Alfred Bernhard Nobel (2e éd.). Stockholm : Norstedts.

Kylhammar, M. (2004), "Sveriges andra stormaktstid ", in Den tidlöse modernisten, Stockholm : Carlssons : 99-I I 8. Traduction française de Battail, M. \& J.-F. (2009), Le Moderniste intemporel. Paris : L'Harmattan.

Mallet, P. H. (1756), Monuments de la Mythologie et de la Poésie des Celtes et particulièrement des Anciens Scandinaves, Copenhague.

Polhem, C. ( I76I), Patriotiska testamentet (posthume).

Tegnér, E. (I8I7), «Språken ». Mindre dikter. Project Runeberg http:// runeberg.org/tegner/0 57.htlm

Östman, M. \& H. (2005), Au champ d'Apollon. Ecrits d'expression française produits en Suède (1550-2006). Stockholm : Kungl. Vitterhetsakademien.

Östman, M. \& H. (2006), "Admiration et aversion. L'attitude des Suédois à l'égard des Français aux XVII et XVIII ${ }^{e}$ siècles ». Études Germaniques 4 (2006) : 229-246. 